\title{
The role of vascular invasion and lymphatic invasion in predicting recurrent thoracic oesophageal squamous cell carcinoma
}

Yu Ma ${ }^{1+}, \mathrm{Xi} \mathrm{Yao}^{2 \dagger}$, Zhenzhen $\mathrm{Li}^{1 \dagger}$, Jie Chen ${ }^{1}$, Wensheng $\mathrm{Li}^{1}$, Hongtao Wang ${ }^{3}$, Lanjun Zhang ${ }^{4^{*}}$ and Jianfei Zhu ${ }^{3^{*}}$

\begin{abstract}
Background: Numerous studies have addressed lymphovascular invasion (LVI) in patients with thoracic oesophageal squamous cell carcinoma (ESCC); however, little is known about the individual roles of lymphatic invasion (LI) and vascular invasion $(\mathrm{VI})$. We aimed to analyse the prognostic significance of $\mathrm{LI}$ and $\mathrm{VI}$ in patients with thoracic ESCC from a single centre.
\end{abstract}

Methods: This retrospective study included 396 patients with thoracic ESCC who underwent oesophagectomy and lymphadenectomy in our hospital. The relationship between LI, VI and the other clinical features was analysed, and disease-free survival (DFS) was calculated. Survival analysis was performed by univariate and multivariate statistics.

Results: Briefly, VI and LI were present in 25.8\% (102 of 396) and 23.7\% (94 of 396) of ESCC patients, respectively, with 9.15\% patients presenting both $\mathrm{LI}$ and $\mathrm{Vl}$; the remaining patients did not present $\mathrm{LI}$ or $\mathrm{VI}$. We found that $\mathrm{LI}$ was significantly associated with pN stage $(P<0.001)$ and pTNM stage $(P<0.001)$, and similar results were found in $\mathrm{Vl}$. Moreover, survival analysis showed that pT stage $(P<0.001)$, pN stage $(P=0.001)$, pTNM stage $(p<0.001), \mathrm{VI}(P=0.001)$ and LI $(P<0.001)$ were associated with DFS in ESCC. Furthermore, multivariate analysis suggested that $\mathrm{pT}$ stage $(\mathrm{RR}=1.4, P$ $=0.032)$, $p N$ stage $(R R=1.9, P<0.001)$ and $L I(R R=1.5, P=0.008)$ were independent predictive factors for $D F S$. Finally, relapse was observed in 110 patients (lymph node metastasis, 78 and distant, 32) and 147 patients with cancerrelated deaths. Subanalysis showed that LI-positive patients had higher lymph node metastasis, although there was no significant difference ( $32.1 \%$ vs. $15.6 \%, P=0.100$ ).

Conclusions: $\mathrm{LI}$ and VI were common in ESCC; they were all survival predictors for patients with ESCC, and LI was independent. Patients with positive LI were more likely to suffer lymph node metastasis.

Keywords: Oesophageal squamous cell carcinoma, Lymphatic invasion, Vascular invasion, Disease-free survival, Lymph node metastasis

*Correspondence: zhujianfei718@163.com; zhanglj@sysucc.org.cn

${ }^{\dagger}$ Yu Ma, Xi Yao and Zhenzhen Li contributed equally as first authors to this work.

${ }^{3}$ Department of Thoracic Surgery, Shaanxi Provincial People's Hospital, No. 256 Youyi Road West, Xi'an 710068, Shaanxi, People's Republic of China

${ }^{4}$ Department of Thoracic Surgery, Sun Yat-sen University Cancer Center, No. 561 Dongfeng Road East, Guangzhou 510060, Guangdong, People's Republic of China

Full list of author information is available at the end of the article

\section{Introduction}

Worldwide, oesophageal cancer is the seventh most widespread cancer and constitutes the sixth leading cause of cancer death, accounting for $5.3 \%$ of all global cancer deaths [1]. Although some studies have indicated an incidence reduction in the last few decades, oesophageal cancer is a significant public health burden in China [2]. Recently, the rate of superficial oesophageal squamous cell carcinoma has been increasing because of advances in endoscopic 


\section{Patients with ESCC were underwent esophagectomy and lymphadenectomy (Jan 2013-Oct 2017) $\mathrm{N}=\mathbf{5 4 2}$}

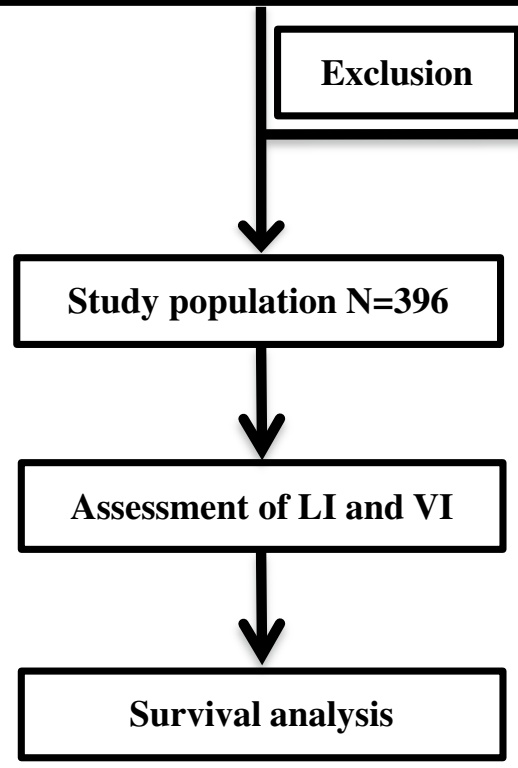

Fig. 1 The flow diagram of this study

diagnosis and treatment; even so, the prognosis remains poor [3, 4]. Several studies have evaluated risk factors for cancer recurrence and survival. Age, tumour length, tumour width, TNM staging, surgical approach, perineural invasion and lymphovascular invasion were significantly associated with locoregional recurrence and distant metastasis after oesophagectomy [5-7]. Moreover, lymphovascular invasion (LVI) has been reported to be a critical pathologic feature of metastasis in urothelial carcinoma, colorectal cancer and several kinds of solid carcinoma [8-11]. Although the staging guidelines of the American Joint Cancer Committee on Cancer mandated distinguishing lymphatic and vascular invasion (LI vs. VI) as early as 2005, these guidelines lack a routine standard and objective assessment method to reliably differentiate them [12]. Some studies have reported that immunohistochemistry (IHC) staining appears more reliable to distinguish lymphatic and vascular invasion than haematoxylin and eosin (H\&E) staining [13-15]. Previous studies focused on LI and VI together in patients with thoracic oesophageal squamous cell carcinoma (ESCC); however, the data on the individual role of LI and VI were limited. The aim of the present study was to analyse the individual influence of LI and VI on the prognosis of ESCC.

\section{Methods}

\section{Patients selection}

A retrospective study was performed on 396 patients who underwent oesophagectomy and lymphadenectomy in Shaanxi Provincial People's Hospital between Jan.2013 and Oct. 2017 (Fig. 1). The patients included in the current study met the following criteria: (1) diagnosis of squamous cell carcinoma; (2) complete resection and regular follow-up; (3) no neoadjuvant therapy; (4) no distant metastasis; (5) no other malignant cancer; and (6) immunohistochemical staining of tissues during diagnosis. The patients were followed up until October 2020 by systematic physical examination and standard laboratory screening every 6 months to evaluate tumour recurrence and metastasis.

\section{Pathological evaluation}

All surgical specimens were processed according to standard pathological procedures. Briefly, the gross specimens and lymph nodes were fixed in $10 \%$ neutral buffered formalin immediately after collection and subsequently embedded in paraffin. Serial sections at $4 \mu \mathrm{m}$ thickness were stained with H\&E. Pathological staging was based on the eighth edition of the American Joint Committee on Cancer TNM staging system [16]. All pathologists were blinded to the clinical outcomes. 

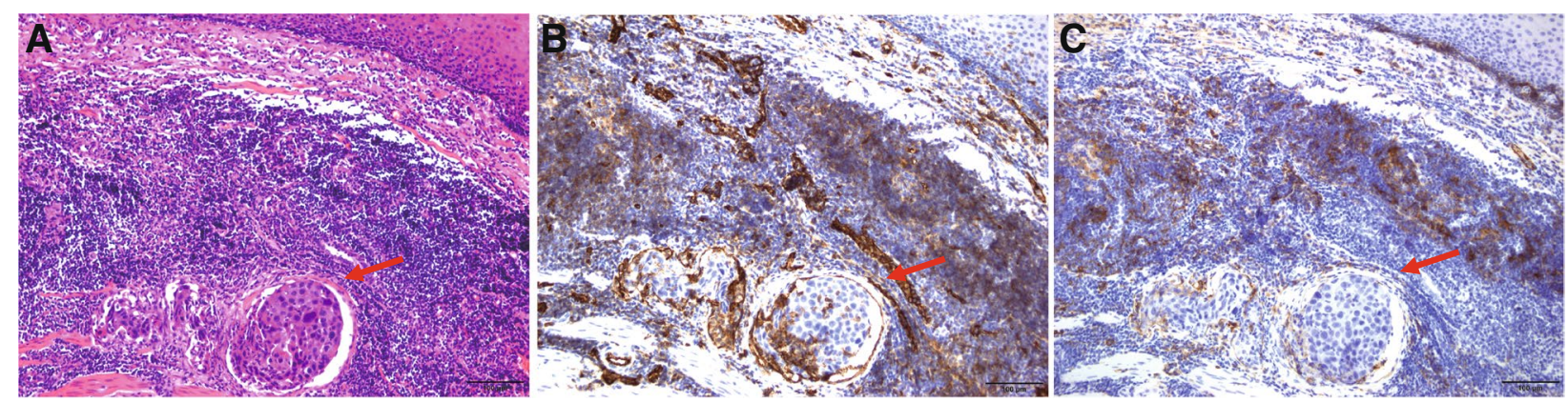

Fig. 2 Representative histology of vascular invasion in oesophageal squamous cell carcinoma. A H\&E staining of conspicuous carcinoma emboli in vascular space (red arrow). B Vascular vessel stained positive for CD31. C Vascular vessel stained negative for D2-40

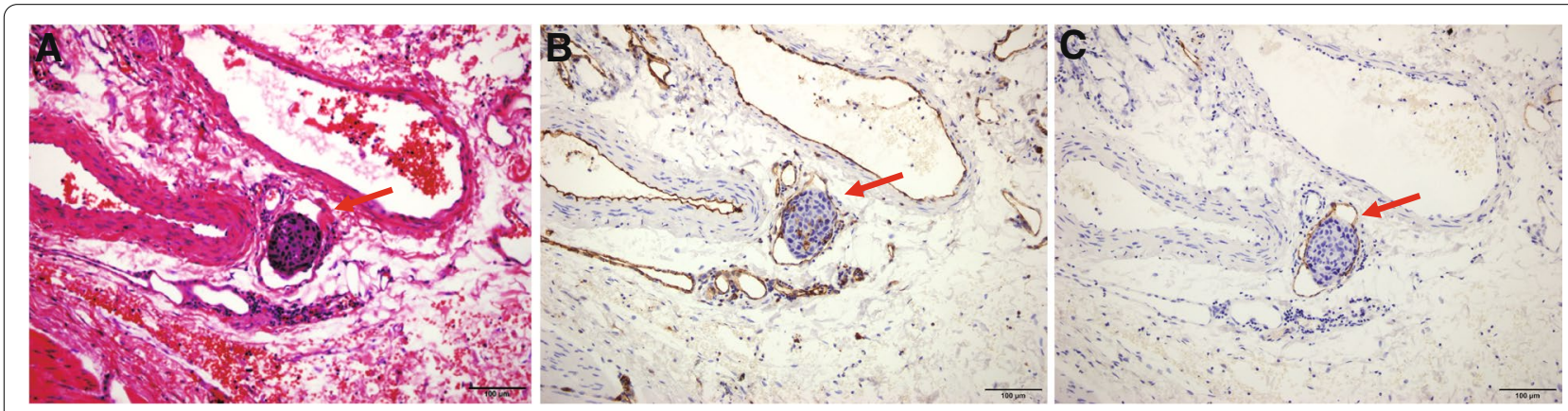

Fig. 3 Representative histology of lymphatic invasion in oesophageal squamous cell carcinoma. A H\&E staining of conspicuous carcinoma emboli in lymphatic vessel space (red arrows). B Lymphatic vessel stained positive for CD31. C Lymphatic vessel stained positive for D2-40

\section{Immunohistochemical staining}

Two consecutive oesophageal carcinoma sections from each patient were stained with monoclonal antibodies against CD31 and D2-40 (ready-to-use, Maixin, China) to assess blood vessels and lymphatic vessels, respectively. Immunohistochemical staining was performed by using an automated immunostainer and an Ultra View Universal DAB (3,3'-diaminobenzidine) Detection Kit (Ventana Medical Systems, Inc. Tucson, AZ, USA). Two full sections of tonsil tissue were used as positive and negative controls for each antibody. All microscopic analyses were carried out using a light microscope (Zeiss, Germany).

\section{Assessment of $\mathrm{LI}$ and $\mathrm{VI}$}

Previous studies have confirmed that neoadjuvant therapy can affect the evaluation of LVI after oesophagectomy $[4,17]$, so we excluded all the specimens of these patients receiving neoadjuvant therapy. $H \& E$ staining of LVI sections was reviewed blinded to the pathological report and evaluated by the attending pathologists. For the assessment of LI and VI, separate sections from the same paraffin block were stained with CD31 and D2-40. The peritumoural and invasive fronts and intratumoural areas were counted. LVI on H\&E-stained sections was identified as the presence of tumour cell emboli within the endothelium-lined spaces. VI was counted only when tumour cells were positive for CD31 and negative for D2-40 (Fig. 2). As CD31 can also stain part of the lymphatic vessels, LI was defined as the lumen where the tumour embolus was located and positively stained with CD31 and D2-40 at the same time (Fig. 3). All specimens were randomly chosen and observed by the different pathologists who were blinded to the previous results. Any inconsistencies were re-evaluated by all the pathologists until a consensus was reached.

\section{Statistical analysis}

The correlation between LVI and clinicopathological features was tested by chi-square test. The KaplanMeier method was used to calculate disease-free survival (DFS), and differences were assessed with the log-rank statistic. All statistically significant prognostic factors identified in the univariate analysis were included in the Cox regression multivariate analysis. $P$ value $<0.05$ was considered to indicate statistical significance. Analyses were performed with SPSS (version 22.0). 


\section{Results}

\section{Clinical and pathological characteristics of included} patients

The median age of the patients was 65 years (range: 42 to 87 ), and $73.2 \%$ of patients were men (290/396). In total, VI was present in $25.8 \%$ of patients (102 of 396), and LI was present in $23.7 \%$ of patients (94 of 396). Among the above patients, 36 patients presented both LI and VI (9.1\%), while the remaining patients did not present LI or VI. The relationships between lymphatic/ vascular invasion and clinicopathological characteristics are listed in Table 1 . VI was significantly associated with pT stage $(P=0.013), \mathrm{pN}$ stage $(P<0.001)$ and pTNM stage $(P<0.001)$, but not with gender, age, smoking status, tumour location, tumour length, surgical approach or differentiation. The only difference between LI and VI was that LI was not associated with pT stage $(P=0.376)$. In detail, LI was present in $20.8 \%$

Table 1 The relationship between lymphovascular invasion and clinicopathological features of oesophageal squamous cell carcinoma

\begin{tabular}{|c|c|c|c|c|c|}
\hline Variable & $\begin{array}{l}\text { Total } \\
396\end{array}$ & $\begin{array}{l}\text { Vascular invasion } \\
\text { Presence(\%) }\end{array}$ & $P$ value & $\begin{array}{l}\text { Lymphatic invasion } \\
\text { Presence(\%) }\end{array}$ & $P$ value \\
\hline Gender & & & 0.437 & & 0.427 \\
\hline Male & 290 & 78 (26.9) & & $72(24.8)$ & \\
\hline Female & 106 & $24(22.6)$ & & $22(20.8)$ & \\
\hline Age & & & 0.766 & & 0.759 \\
\hline$<65$ years & 325 & 85 (26.2) & & $76(23.3)$ & \\
\hline$\geq 65$ years & 71 & $17(23.9)$ & & $18(25.4)$ & \\
\hline Smoking status & & & 0.630 & & 0.537 \\
\hline Non-smoker & 138 & $33(23.9)$ & & $30(21.7)$ & \\
\hline Smoker & 258 & $69(26.7)$ & & $64(24.8)$ & \\
\hline Tumour location & & & 0.347 & & 0.671 \\
\hline Upper & 35 & $7(20.0)$ & & $8(22.9)$ & \\
\hline Middle & 253 & $62(24.5)$ & & $57(22.5)$ & \\
\hline Lower & 108 & $33(30.6)$ & & 29 (26.9) & \\
\hline Tumour length & & & 1.000 & & 0.637 \\
\hline$\leq 4.0 \mathrm{~cm}$ & 199 & $51(25.6)$ & & 45 (22.6) & \\
\hline$>4.0 \mathrm{~cm}$ & 197 & $51(25.9)$ & & $49(24.9)$ & \\
\hline Surgical approach & & & 1.000 & & 0.897 \\
\hline Right incision & 118 & $30(25.4)$ & & 27 (22.9) & \\
\hline Left incision & 278 & 72 (25.9) & & $67(24.1)$ & \\
\hline Differentiation & & & 0.274 & & 0.997 \\
\hline G1 & 119 & $37(31.1)$ & & $28(23.5)$ & \\
\hline G2 & 184 & $44(23.9)$ & & $44(23.9)$ & \\
\hline G3 & 93 & $21(22.6)$ & & $22(23.7)$ & \\
\hline pTstage & & & 0.013 & & 0.376 \\
\hline $\mathrm{T} 1+\mathrm{T} 2$ & 125 & $22(17.6)$ & & $26(20.8)$ & \\
\hline $\mathrm{T} 3+\mathrm{T} 4$ & 271 & $80(29.5)$ & & $68(25.1)$ & \\
\hline p N stage & & & $<0.001$ & & $<0.001$ \\
\hline No & 210 & $32(15.2)$ & & $33(15.7)$ & \\
\hline $\mathrm{N} 1+\mathrm{N} 2+\mathrm{N} 3$ & 186 & 70 (37.6) & & $61(32.8)$ & \\
\hline pTNM stage & & & $<0.001$ & & $<0.001$ \\
\hline I stage & 49 & $4(8.2)$ & & $6(12.2)$ & \\
\hline II stage & 164 & $29(54.9)$ & & $25(15.2)$ & \\
\hline III stage & 170 & $60(35.3)$ & & $55(32.4)$ & \\
\hline IV stage & 13 & $9(69.2)$ & & $8(61.5)$ & \\
\hline Relapse type & & & 0.816 & & 0.100 \\
\hline Lymph node recurrence & 78 & $23(29.5)$ & & $25(32.1)$ & \\
\hline Metastasis & 32 & $8(25.0)$ & & $5(15.6)$ & \\
\hline
\end{tabular}


(26/125) of patients in $\mathrm{T} 1+\mathrm{T} 2$ and $25.1 \%(68 / 271)$ of patients in $\mathrm{T} 3+\mathrm{T} 4$.

\section{Survival analysis}

In Table 2, the predictors for DFS of patients with ESCC by univariate analysis are listed. Briefly, $\mathrm{pT}$ stage $(P<0.001)$, pN stage $(P=0.01)$, pTNM stage $(P<0.001)$, VI $(P=0.01)$ and LI $(P<0.001)$ were associated with DFS in ESCC. Among all patients, the prognosis of patients was the worst in those with double positivity (VI and LI) and the best for patients with double negativity (VI and LI); however, there was no significant difference in individual LI positivity and VI positivity (45.1 months vs. 27.2 months vs. 24.5 months vs. 11.6 months, respectively; $P<0.001$ ) (Fig. 4). When reviewing DFS, both LI and VI were predictors of survival (LI: DFS 41.0 months vs. 18.6 months, $P<0.01$; VI: DFS 41.8 months vs. 21.0 months, $P=0.001$ ) (Fig. 5).

\section{Independent factors affect DFS of ESCC}

A multivariate analysis using the Cox proportional hazard model for all the patients showed that $\mathrm{pT}$ stage $(\mathrm{RR}=1.4, P=0.032), \mathrm{pN}$ stage $(\mathrm{RR}=1.9, P<0.001)$ and $\mathrm{LI}$ ( $\mathrm{RR}=1.5, P=0.008$ ) were predictive factors for prognosis with regard to DFS. Meanwhile, VI $(\mathrm{RR}=1.3, P=0.086)$ was not a prognostic factor. This suggests that individual LI, but not LVI or individual VI, is a dependent prognostic factor for ESCC (Table 3).

\section{Recurrence and metastasis modes of ESCC}

The median follow-up time was 57.3 months (range: 4.9-94.2). Loss to follow-up was 7.8\% (31/296). Relapse was observed in 110 patients (lymph node metastasis 78 or distant 32); 141 patients were still alive at the last follow-up, and 147 patients had cancer-related deaths. Subanalysis showed that LI-positive patients had higher lymph node metastasis, although there was no significant difference $(32.1 \%$ vs. $15.6 \%, P=0.100)$. The data are summarized in Table 1.

\section{Discussion}

Several previous studies have shown that LVI influences the prognosis of ESCC [18-20]. Hsu CP et al. [4] considered that the prognostic impact of LVI was primarily in the subgroup of node-negative patients who received primary oesophagectomy. Similarly, Huang et al. [21] indicated that in patients who underwent primary oesophagectomy, LVI was associated with poor disease-specific survival or disease-free survival, and they believed that LVI may precede or occur concurrently with lymph node metastasis. In the current study, the results were in accordance with their findings, and both LI and VI were predictors of survival.
Table 2 Predictors for disease-free survival in oesophageal squamous cell carcinoma by univariate analysis

\begin{tabular}{|c|c|c|c|}
\hline Variable & Median & $\begin{array}{l}\text { Disease-free } \\
\text { survival (months) } \\
95 \% \mathrm{Cl}\end{array}$ & $P$ value \\
\hline Gender & & & 0.029 \\
\hline Male & 29.2 & $23.1-35.3$ & \\
\hline Female & 42.9 & $22.4-63.3$ & \\
\hline Age & & & 0.608 \\
\hline$<65$ years & 34.2 & $26.6-41.9$ & \\
\hline$\geq 65$ years & 27.2 & $20.5-33.9$ & \\
\hline Smoking status & & & 0.064 \\
\hline Non-smoker & 43.7 & $20.9-66.4$ & \\
\hline Smoker & 28.7 & $22.6-34.8$ & \\
\hline Tumour location & & & 0.379 \\
\hline Upper & 27.1 & $20.7-33.5$ & \\
\hline Middle & 39.2 & $31.1-47.2$ & \\
\hline Lower & 27.2 & 20.9-33.5 & \\
\hline Tumour length & & & 0.003 \\
\hline$\leq 4.0 \mathrm{~cm}$ & 42.9 & $34.4-51.3$ & \\
\hline$>4.0 \mathrm{~cm}$ & 27.4 & $23.0-31.8$ & \\
\hline Surgical approach & & & 0.734 \\
\hline Right incision & 27.2 & $19.3-35.0$ & \\
\hline Left incision & 35.3 & $27.7-42.8$ & \\
\hline Differentiation & & & 0.763 \\
\hline G1 & 30.0 & $19.7-40.3$ & \\
\hline $\mathrm{G} 2$ & 32.8 & $22.0-43.6$ & \\
\hline G3 & 35.5 & $21.0-49.8$ & \\
\hline pTstage & & & $<0.001$ \\
\hline $\mathrm{T} 1+\mathrm{T} 2$ & 56.3 & $28.3-84.3$ & \\
\hline $\mathrm{T} 3+\mathrm{T} 4$ & 27.1 & $22.6-31.5$ & \\
\hline p N stage & & & $<0.001$ \\
\hline No & 68.5 & 45.4-91.6 & \\
\hline $\mathrm{N} 1+\mathrm{N} 2+\mathrm{N} 3$ & 19.7 & $13.9-25.5$ & \\
\hline Adjuvant therapy & & & 0.570 \\
\hline With & 38.8 & $29.4-48.2$ & \\
\hline Without & 27.8 & $22.4-33.1$ & \\
\hline pTNM stage & & & $<0.001$ \\
\hline I stage & 71.4 & 43.9-98.8 & \\
\hline II stage & 56.7 & $31.1-82.3$ & \\
\hline III stage & 21.6 & $15.7-27.5$ & \\
\hline IV stage & 10.8 & $4.9-16.6$ & \\
\hline Vascular invasion & & & 0.001 \\
\hline Presence & 21.0 & $15.5-26.5$ & \\
\hline No & 41.8 & $32.1-51.6$ & \\
\hline Lymphatic invasion & & & $<0.001$ \\
\hline Presence & 18.6 & $11.1-26.1$ & \\
\hline No & 41.0 & $31.8-50.2$ & \\
\hline
\end{tabular}




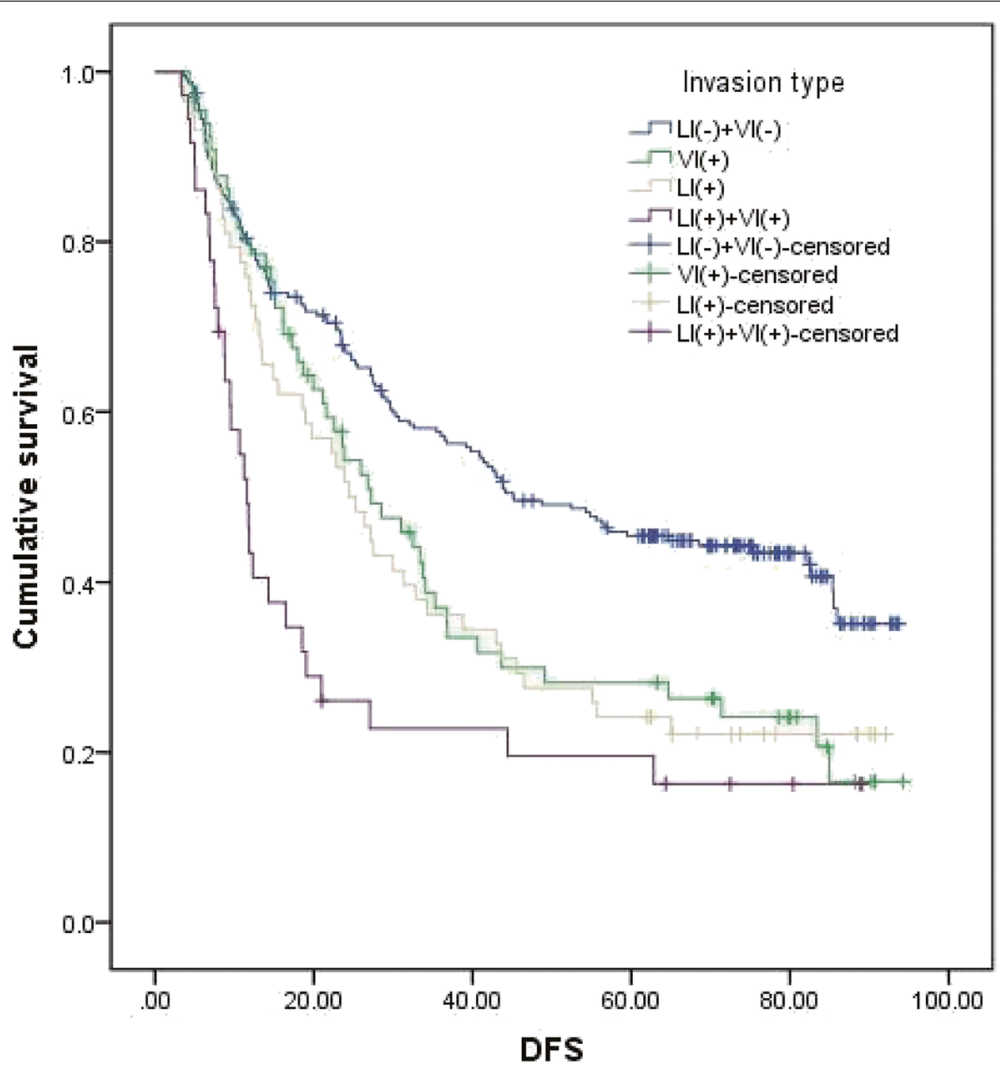

Fig. 4 Disease-free survival according to the type of lymphovascular invasion
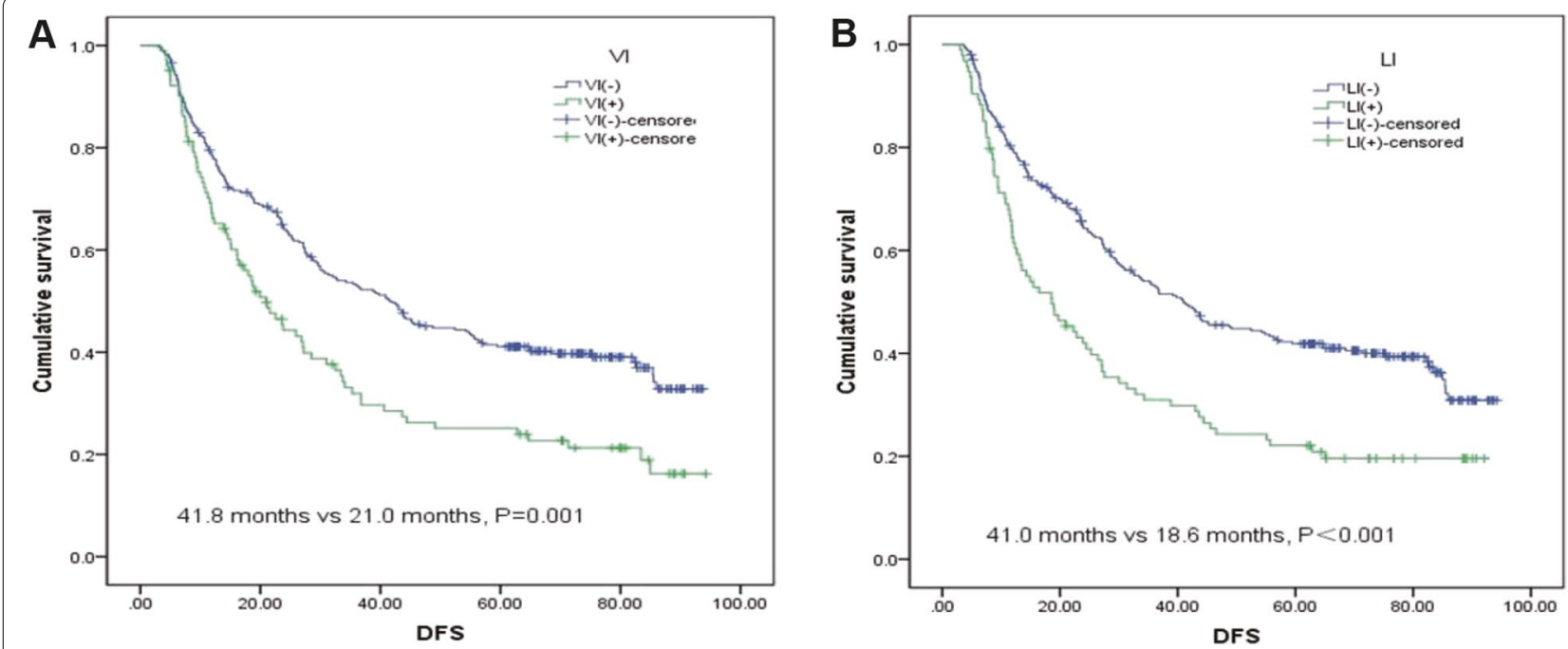

Fig. 5 Disease-free survival according to the individual roles of vascular invasion and lymphatic invasion. A Disease-free survival according to the individual role of vascular invasion. B DFS according to the individual role of lymphatic invasion

Moreover, most of the previous studies combined LI and VI together and investigated the effects of prognosis on lymphatic and vascular invasion together [22-24].
Individual studies of LI and VI are scattered. A previous study suggested that angiogenesis and lymphatic vessel formation play different roles in the early stages of 
Table 3 Independent factors affecting disease-free survival in oesophageal squamous cell carcinoma by multivariate analysis

\begin{tabular}{llll}
\hline Variable & RR & $\begin{array}{l}\text { Disease-free } \\
\text { survival } \\
\mathbf{9 5 \%} \mathbf{C l}\end{array}$ & $\boldsymbol{P}$ value \\
\hline Gender: female/male & 0.8 & $0.6-1.1$ & 0.260 \\
Tumour length: $\leq 4.0 \mathrm{~cm} />4.0 \mathrm{~cm}$ & 1.2 & $0.9-1.5$ & 0.171 \\
Differentiation:G1/G2/G3 & 1.0 & $0.8-1.1$ & 0.595 \\
p T stage:T1+T2/T3+T4 & 1.4 & $1.0-1.8$ & 0.032 \\
p N stage:N0/N1+N2+N3 & 1.9 & $1.5-2.5$ & $<0.001$ \\
Vascular invasion: no/presence & 1.3 & $1.0-1.7$ & 0.086 \\
Lymphatic invasion: no/presence & 1.5 & $1.1-1.9$ & 0.008 \\
\hline
\end{tabular}

tumour formation, and they found that lymphangiogenesis may promote the initial metastatic process of nonseminomatous testicular germ cell tumours rather than angiogenesis and blood vascular invasion [25]. Additionally, Spoerl S et al. [26] reported significantly lower OS and RFS in patients with LI (OS: 41.1\%, RFS: $38.3 \%$ ) in contrast to patients with LI-negative oral squamous carcinoma (OS: $66.8 \%$, RFS: $59.7 .7 \%, P<0.001$ ). However, there were limited similar studies on ESCC. In the current study, LI and VI were predictors of survival for patients with ESCC, and LI was an independent factor, indicating that LI might play an important role in ESCC.

The Japan Esophageal Society guideline of 2017 recommended that endoscopic resection (ER) can be applied for lesions that infiltrate the muscularis mucosae or inner submucosa (T1b-SM1), but the risk of lymph node metastasis still exists for these cases. Deeper superficial carcinomas (T1b-SM2 and T1b-SM3) should not be treated with endoscopy alone due to the high rates of metastasis [27, 28]. Different observations have been made in the literature concerning the possible reasons. J. Oguma et al. [29] hypothesized that lymphatic invasion occurs first during invasion from the muscularis mucosae to the upper layer of the submucosa, and venous invasion may occur after lymphatic invasion as the tumour invades deeper layers of the submucosa. The mechanical stress generated by proliferating tumour cells and high interstitial fluid pressure may affect intratumour vascular function [25]. LI seems to be more affected by these processes than VI, which might be due to the thin endothelial wall and missing basal membrane of the lymph vessels $[25,30]$.

In a review of previous literature, the majority of methods for identifying LVI were based on H\&E staining. However, some studies have used special biomarkers for the lymphatic and vascular endothelia to accurately detect and distinguish lymphatic and vascular invasion. Faiz et al. [31] identified the type of vascular invasion of tumour cells by performing additional Elastica van Gieson staining to confirm or exclude the presence of extramural venous invasion. In this study, the recognition of LI and VI was based on immunohistochemical staining with an anti-D2-40 antibody for lymph vessels and an antiCD31 antibody for blood vessels. In some cases diagnosed as LVI negative on H\&E staining, immunostaining discerned the retraction artefacts and confirmed the existence of lymphatic vessels when a tumour embolus completely obliterated the lumen of the lymphatic channel [32, 33]. In our experience, immunohistochemistry has been routinely used in the Pathology Department quickly and effectively at our institution.

Postoperative recurrence of oesophageal cancer is also a concern. Our team found that local recurrence was twice as common as distant recurrence in the first 3 years after oesophagectomy, and the type of local recurrence was mainly lymph node metastasis [34]. Yang and his colleague conducted a meta-analysis of the relationship of LVI with lymph node metastasis and prognosis in superficial oesophageal cancer and concluded that LVI plays an important role in the prognosis of lymph node metastasis in superficial oesophageal cancer [20]. In this study, LI could not only assess preoperative lymph node metastasis but also predict postoperative local lymph node recurrence.

With the release of a series of clinical study results [35-37], neoadjuvant therapy (neoadjuvant chemotherapy and neoadjuvant chemoradiation) is recommended by many guidelines as induction therapy for oesophageal cancer, and our clinical practice is also recommended in accordance with relevant guidelines. A meta-analysis of different neoadjuvant treatments for oesophageal cancer included 25 randomized trials involving 5272 patients, its result showed that compared with chemotherapy, neoadjuvant concurrent chemoradiation has obvious advantages in the survival of surgical treatment of resectable oesophageal cancer [38]. Our study was to explore the effect of LVI of thoracic oesophageal cancer on the prognosis of patients. Therefore, all patients receiving neoadjuvant therapy were excluded from this study, which is consistent with previous related studies [21, 39].

There are some limitations to the present study that need to be addressed. First, in our retrospective study, an inherent bias cannot be excluded. Second, we only analysed the overall positive or negative results in LI and VI, and peritumoural, invasive front and intratumoural areas should be investigated in future research. Moreover, although we use various methods to follow up patients, $7.8 \%$ of patients are still lost in the process. Nevertheless, in our study, 147 patients died of cancer but showed no clear signs of metastasis and recurrence, which might have skewed the survival analysis. Prospective studies 
are necessary in future work, which could potentially guide new staging methods and treatment principles of ESCC.

\section{Conclusion}

We concluded that LVI is an important supplement to the TNM staging of ESCC and that LI and VI should be evaluated separately. LI could not only assess preoperative lymph node metastasis but also predict postoperative local lymph node recurrence.

\section{Acknowledgements}

Not applicable.

\section{Authors' contributions}

Jianfei Zhu, Yu Ma and Lanjun Zhang participated in study design and study conception; Jianfei Zhu and Yu Ma performed statistical analysis; Hongtao Wang and Jianfei Zhu performed the surgery; Yu Ma, Zhenzhen Li, Jie Chen and Wensheng Li performed pathological diagnosis; Jianfei Zhu and Xi Yao recruited patients. Jianfei Zhu, Yu Ma and Xi Yao drafted the manuscript. All authors provided critical review of the manuscript and approved the final draft for publication.

\section{Funding}

This research was supported by grants from the Wu Jieping Medical Foundation (320.6750.17527).

\section{Availability of data and materials}

Not applicable.

\section{Declarations}

\section{Ethics approval and consent to participate}

This study was approved by the ethics committee of the Shaanxi Provincial People's Hospital (N0.20210803).

\section{Consent for publication}

Not applicable.

\section{Competing interests}

The authors declare that they have no competing interests.

\section{Author details}

${ }^{1}$ Department of Pathology, Shaanxi Provincial People's Hospital, No. 256 Youyi Road West, Xi'an 710068, Shaanxi, People's Republic of China. ${ }^{2}$ Department of Anesthesiology, Shaanxi Provincial People's Hospital, No. 256 Youyi Road West, Xi'an 710068, Shaanxi, People's Republic of China. ${ }^{3}$ Department of Thoracic Surgery, Shaanxi Provincial People's Hospital, No. 256 Youyi Road West, Xi'an 710068, Shaanxi, People's Republic of China. ${ }^{4}$ Department of Thoracic Surgery, Sun Yat-sen University Cancer Center, No. 561 Dongfeng Road East, Guangzhou 510060, Guangdong, People's Republic of China.

Received: 12 August 2021 Accepted: 25 November 2021

Published online: 10 January 2022

\section{References}

1. Sung H, Ferlay J, Siegel RL, Laversanne M, Soerjomataram I, Jemal A, et al. Global Cancer Statistics 2020: GLOBOCAN estimates of incidence and mortality worldwide for 36 cancers in 185 countries. CA Cancer J Clin. 2021;71:209-49.

2. Yang J, Liu X, Cao S, Dong X, Rao S, Cai K. Understanding esophageal cancer: the challenges and opportunities for the next decade. Front Oncol. 2020;10:1727.

3. Parry K, Visser E, van Rossum PS, Mohammad NH, Ruurda JP, van Hillegersberg R. Prognosis and treatment after diagnosis of recurrent esophageal carcinoma following esophagectomy with curative intent. Ann Surg Oncol. 2015;22(Suppl 3):S1292-300.
4. Hsu CP, Chuang CY, Hsu PK, Chien LI, Lin CH, Yeh YC, et al. Lymphovascular invasion as the major prognostic factor in node-negative esophageal cancer after primary esophagectomy. J Gastrointest Surg. 2020;24:1459-68.

5. Zhang WY, Chen XX, Chen WH, Zhang H, Zou CL. Nomograms for predicting risk of locoregional recurrence and distant metastases for esophageal cancer patients after radical esophagectomy. BMC Cancer. 2018;18:879.

6. Lam KO, So TH, Kong FS. Predicting liver and lung metastasis in esophageal cancer: does the site of primary tumor really matter? J Thorac Dis. 2020;12:2996-9.

7. Liu S, Anfossi S, Qiu B, Zheng Y, Cai M, Fu J, et al. Prognostic factors for locoregional recurrence in patients with thoracic esophageal squamous cell carcinoma treated with radical two-field lymph node dissection: results from long-term follow-up. Ann Surg Oncol. 2017;24:966-73.

8. Kikuchi E, Margulis V, Karakiewicz PI, Roscigno M, Mikami S, Lotan Y, et al. Lymphovascular invasion predicts clinical outcomes in patients with node-negative upper tract urothelial carcinoma. J Clin Oncol. 2009;27:612-8

9. Cheng SP, Lee JJ, Chien MN, Kuo CY, Jhuang JY, Liu CL. Lymphovascular invasion of papillary thyroid carcinoma revisited in the era of active surveillance. Eur J Surg Oncol. 2020;46:1814-9.

10. Roma AA, Park KJ, Xie H, De Vivar AD, Alvarado-Cabrero I, Rutgers J, et al. Role of lymphovascular invasion in pattern c invasive endocervical adenocarcinoma. Am J Surg Pathol. 2017;41:1205-11.

11. Al-Sukhni E, Attwood K, Gabriel EM, LeVea CM, Kanehira K, Nurkin SJ. Lymphovascular and perineural invasion are associated with poor prognostic features and outcomes in colorectal cancer: a retrospective cohort study. Int J Surg. 2017;37:42-9.

12. Gujam FJ, Going JJ, Mohammed ZM, Orange C, Edwards J, McMillan DC. Immunohistochemical detection improves the prognostic value of lymphatic and blood vessel invasion in primary ductal breast cancer. BMC Cancer. 2014;14:676.

13. van Wyk HC, Roxburgh CS, Horgan PG, Foulis AF, McMillan DC. The detection and role of lymphatic and blood vessel invasion in predicting survival in patients with node negative operable primary colorectal cancer. Crit Rev Oncol Hematol. 2014;90:77-90.

14. Gujam FJ, Going JJ, Edwards J, Mohammed ZM, McMillan DC. The role of lymphatic and blood vessel invasion in predicting survival and methods of detection in patients with primary operable breast cancer. Crit Rev Oncol Hematol. 2014;89:231-41.

15. Hosono I, Miyahara R, Furukawa K, Funasaka K, Sawada T, Maeda K, et al. Use of Immunostaining for the diagnosis of Lymphovascular invasion in superficial Barrett's esophageal adenocarcinoma. BMC Gastroenterol. 2020;20:175.

16. Donohoe CL, Phillips AW. Cancer of the esophagus and esophagogastric junction: an 8(th) edition staging primer. J Thorac Dis. 2017;9:E282-4.

17. Kurokawa T, Hamai Y, Emi M, Ibuki Y, Yoshikawa T, Ohsawa M, et al. Risk factors for recurrence in esophageal squamous cell carcinoma without pathological complete response after trimodal therapy. Anticancer Res. 2020;40:4387-94

18. Lagarde SM, Phillips AW, Navidi M, Disep B, Immanuel A, Griffin SM. The presence of lymphovascular and perineural infiltration after neoadjuvant therapy and oesophagectomy identifies patients at high risk for recurrence. Br J Cancer. 2015;113:1427-33.

19. Hsu PK, Chien LI, Wang LC, Chou TY. Lymphovascular invasion and extracapsular invasion are risk factors for distant recurrence after preoperative chemoradiotherapy and oesophagectomy in patients with oesophageal squamous cell carcinoma. Eur J Cardiothorac Surg. 2017;51:1188-94.

20. Yang J, Lu Z, Li L, Li Y, Tan Y, Zhang D, et al. Relationship of lymphovascular invasion with lymph node metastasis and prognosis in superficial esophageal carcinoma: systematic review and meta-analysis. BMC Cancer. 2020;20:176.

21. Huang Q, Luo K, Chen C, Wang G, Jin J, Kong M, et al. Identification and validation of lymphovascular invasion as a prognostic and staging factor in node-negative esophageal squamous cell carcinoma. J Thorac Oncol. 2016;11:583-92.

22. Yu B, Park JY, Park KB, Kwon OK, Lee SS, Chung HY. Prognostic factors in stage IB gastric cancer after surgical resection. J Gastric Cancer. 2020;20:328-36.

23. Wang S, Zhang B, Qian J, Qiao R, Xu J, Zhang L, et al. Proposal on incorporating lymphovascular invasion as a T-descriptor for stage I lung cancer. Lung Cancer. 2018;125:245-52. 
24. Zhong JW, Yang SX, Chen RP, Zhou YH, Ye MS, Miao L, et al. Prognostic value of lymphovascular invasion in patients with stage III colorectal cancer: a retrospective study. Med Sci Monit. 2019;25:6043-50.

25. Heinzelbecker J, Gross-Weege M, Weiss C, Horner C, Trunk MJ, Erben P, et al. Microvascular invasion of testicular nonseminomatous germ cell tumors: implications of separate evaluation of lymphatic and blood vessels. J Urol. 2014;192:593-9.

26. Spoerl S, Gerken M, Fischer R, Mamilos A, Spoerl S, Wolf S, et al. Lymphatic and vascular invasion in oral squamous cell carcinoma: implications for recurrence and survival in a population-based cohort study. Oral Oncol. 2020;111:105009.

27. Japanese Classification of Esophageal Cancer, 11th Edition: part I. Esophagus-Tokyo. 2017;14:1-36.

28. Japanese Classification of Esophageal Cancer, 11th Edition: part II and III. Esophagus-Tokyo. 2017;14:37-65.

29. Oguma J, Ozawa S, Kazuno A, Yamamoto M, Ninomiya Y, Yatabe K, et al. Prognostic impact of lymphovascular invasion in lymph node-negative superficial esophageal squamous cell carcinoma. Dis Esophagus. 2019;32.

30. Saharinen P, Tammela T, Karkkainen MJ, Alitalo K. Lymphatic vasculature: development, molecular regulation and role in tumor metastasis and inflammation. Trends Immunol. 2004;25:387-95.

31. Faiz Z, Huijgen L, Alqethami HJ, Burgerhof J, Kats-Ugurlu G, Plukker J. Prevalence and prognostic significance of extramural venous invasion in patients with locally advanced esophageal cancer. Ann Surg Oncol. 2018;25:1588-97.

32. Arnaout-Alkarain A, Kahn HJ, Narod SA, Sun PA, Marks AN. Significance of lymph vessel invasion identified by the endothelial lymphatic marker D2-40 in node negative breast cancer. Mod Pathol. 2007;20:183-91.

33. Ukai R, Hashimoto K, Nakayama H, Iwamoto T. Lymphovascular invasion predicts poor prognosis in high-grade pT1 bladder cancer patients who underwent transurethral resection in one piece. Jpn J Clin Oncol. 2017:47:447-52

34. Zhu JF, Feng XY, Zhang XW, Wen YS, Lin P, Cai L, et al. Time distribution of recurrence risk of oesophageal squamous cell carcinoma with complete resection (R0) in a Chinese population. Eur J Cardiothorac Surg. 2015;48:899-905.

35. Hsu PK, Chen HS, Liu CC, Wu SC. Neoadjuvant chemoradiation versus upfront esophagectomy in clinical stage II and III esophageal squamous cell carcinoma. Ann Surg Oncol. 2019;26:506-13.

36. Yang H, Liu H, Chen Y, Zhu C, Fang W, Yu Z, et al. Long-term efficacy of neoadjuvant chemoradiotherapy plus surgery for the treatment of locally advanced esophageal squamous cell carcinoma: the NEOCRTEC5010 randomized clinical trial. JAMA Surg. 2021;156:721-9.

37. Yamagata Y, Saito K, Hirano K, Oya M. Long-term outcomes and safety of radical transmediastinal esophagectomy with preoperative docetaxel, cisplatin, and 5-fluorouracil combination chemotherapy for locally advanced squamous cell carcinoma of the thoracic esophagus. World J Surg Oncol. 2020;18:252.

38. Kumar T, Pai E, Singh R, Francis NJ, Pandey M. Neoadjuvant strategies in resectable carcinoma esophagus: a meta-analysis of randomized trials. World J Surg Oncol. 2020;18:59.

39. Zhang H, Chen X, Wang S, Fan J, Lu L. Poorer prognosis associated with simultaneous lymphatic and vascular invasion in patients with squamous carcinoma of the thoracic oesophagus. Eur J Cardiothorac Surg. 2017;52:378-84.

\section{Publisher's Note}

Springer Nature remains neutral with regard to jurisdictional claims in published maps and institutional affiliations. 